Revista Española de Filosofía Medieval, 2 (1995), pp. 71-78

\title{
LA POLÉMICA DE PEDRO DE ATARRABIA (M. 1347) CON PEDRO AUREOLO (M. 1322) SOBRE LA INTUICIÓN DEL NO-EXISTENTE
}

\author{
Ana Azanza \\ Universidad de Navarra
}

\section{RESUMEN}

Pedro de Atarrabia (m. 1348), filósofo escotista se plantea en una cuestión quodlibetal (c. 1320) la diferencia entre la intuición y la abstracción en lo que se refiere al conocimiento de la existencia Piensa Atarrabia que la abstracción se distingue de la intuición por necesitar dos especies vicarias. Además la intuición precisa de la existencia actual «exterior» del objeto conocido. Atarrabia rechaza la pretensión de su contemporáneo Pedro Aureolo sobre el conocimiento intuitivo de lo no-existente.

\begin{abstract}
Pedro of Atarrabia (d. 1348), scotist philosopher, arrives, through a quodlibetal subject, to the difference between intuition and abstraction, in all that refers to the knowledge of existence. Atarrabia thinks that abstraction needs subordinate «species» while intuition does not need them. He rejects his contemporary, Petrus Aureolus, who pretends that non-existent objects can be known by intuition.
\end{abstract}

\section{AUTORÍA Y CRONOLOGÍA DEL PRIMER QUODLIBETO DE ATARRABIA}

Pedro de Navarra o de Atarrabia, conocido como doctor fundatus, ${ }^{1}$ ha sido objeto de varios interesantes estudios por parte del investigador ya fallecido Pío Sagués. ${ }^{2}$ A él se debe

1 Fraile franciscano, teólogo y consejero de los monarcas navarros. Nació probablemente en Villava, en su término de Atarrabia. Se desconoce la fecha de su nacimiento; debió de ser en el último cuarto del siglo XIII. Estudió en París donde siguió las lecciones de un discípulo de Duns Escoto y obtuvo el grado de maestro en Teología. Vuelto a España, explicó en los centros de estudio franciscanos del reino de Aragón. Fue al menos dos veces provincial del reino de Aragón, y asistió al capítulo general de la Orden, celebrado en Perpiñán en 1331. Los reyes de Navarra le tuvieron en gran estima y le encomendaron misiones delicadas y de gran importancia. También mantuvo estrechas relaciones con Jaime II de Aragón. Su única obra conocida es In Primum Sententiarum Scriptum y se le atribuyen algunas cuestiones quodlibetales que analizaremos seguidamente.

2 Pío Saguiés, El Maestro Pedro de Navarra O.F.M. (1347) "Doctor Fundatus» y su comentario sobre el libro I de las Sentencias, Discurso de recepción en la Academia de Doctores, Madrid, 1966; Id., «Un 
la publicación de la principal obra de Atarrabia conocida hasta hoy, In Primum Sententiarum Scriptum, Madrid, 1966. En el mismo volumen el profesor Pío Sagüés incluyó dos cuestiones quodlibetales inéditas provenientes del manuscrito Vaticano Latino 1.012. Para elaborar este artículo hemos estudiado el contenido de la primera de las dos cuestiones que se encuentra en dicho manuscrito, ff. 92 rb-93 ra. Se titula: Postea quaerebatur utrum cognitio intuitiva et abstractiva distinguantur ab invicem per hoc quod est concernere vel non concernere exsistentiam rei sub rationes obiecti cogniti. Como se ve por el enunciado, el tema central de esta cuestión es el conocimiento abstracto e intuitivo, lo que nos llevó a pensar en el interés de una comparación entre el pensamiento de Atarrabia y el de Pedro Aureolo un autor contemporáneo suyo.

Aunque según numerosos especialistas, como Grabmann, el texto que estudiamos es anónimo, Pío Sagüés demuestra con razones de bastante fundamento que el autor de la cuestión es Pedro de Atarrabia, pues existe un estrecho paralelismo entre ella y el desarrollo que el mismo fray Pedro realiza en el prólogo a su Comentario a las Sentencias. ${ }^{3}$ Creemos, pues, que la autoría de esta obra está clara tras los trabajos de Pío Sagüés. ${ }^{+}$Por otra parte, en el prólogo de este Comentario, tratando acerca del conocimiento abstracto, dice Pedro de Atarrabia: «alias fuit dictum», ${ }^{5}$ lo que lleva a pensar que se está refiriendo a un escrito suyo anterior que bien pudiera ser esta cuestión quodlibet de la que ahora nos ocupamos.

La obra In Primum Sententiarum Scriptum hay que fecharla entre 1320 y 1323. Por tanto, la cuestión sobre la diferencia entre abstracción e intuición debió de ser escrita por Atarrabia antes de 1320. Es posible que se trate de uno de los ejercicios exigidos a los bachilleres en teología, cuando opositaban para alcanzar el grado de maestro, y que posteriormente haya recibido la forma quodlibetal.

Atarrabia fue superior de la provincia franciscana de Aragón, al menos en dos ocasiones. Las fechas de su primer mandato se sitúan entre 1317 y 1320, según lo prueba una serie de cartas escritas por nuestro autor en 1318 y dirigidas al rey Jaime II de Aragón. ${ }^{6}$ Ello podría significar que fray Pedro compuso su obra al menos antes de 1317, porque no es probable que haya podido compaginar el gobierno con una actividad magisterial ordinaria. Guiándonos por estos resultados de la investigación, todo apunta a pensar que Atarrabia estudió en París en la primera década del siglo XIV, con lo que aumentan las

escotista desconocido. El Maestro Pedro de Navarra y el prólogo de su comentario sobre las Sentencias», Verdad y Vida, 24 (1966), pp. 351-354; Id., "Fray Pedro de Atarrabia y Jaime II de Aragón». Archivo IberoAmericano, 27 (1967), pp. 451-460; Id., «¿Una nueva obra del Maestro Pedro de Navarra?», Verdad y Vida, 26 (1968), pp. 263-267; Id., «El códice anónimo Vat. Lat. 5.365 y el Maestro Pedro de Navarra», Verdad y Vida, 28 (1970), pp. 105-107; Id., «Una cuestión inédita del cod. Vat. Lat. 1.012», Estudios Eclesiásticos, 47 (1972), pp. 555-583.

3 Cfr. Pedro de Atarrabia, In Primum Sententiarum Scriptum, edición de Pío Saguiés, Madrid, 1966. Prólogo, Pars 1, q. 2 (n. 45-46 y 50).

4 Verdad y Vida, 26 (1968), p. 265.

5 In Primum Sententiarum Scriptum, Prol., Pars I, q. 2 («III. Responsio ad rationes aliorum», p. 16).

6 Pío Saguiés, «Fray Pedro de Atarrabia y Jaime II de Aragón». 
posibilidades de que asistiera a las lecciones de Juan Duns Escoto, quien fue - como se sabe- el primer gran maestro que se planteó a fondo el tema de la intuición intelectual.

\section{QUÉ DICE ATARRABIA}

Parte de que no se puede conocer intuitivamente lo no existente, porque la intuición versa sobre la existencia. Eso es necesariamente lo que la caracteriza frente a la abstracción. La intuición y la abstracción se distinguen, pues, porque sólo intuitivamente conocemos la existencia. Ésta es la regla gnoseológica general. No obstante, y como veremos después, Atarrabia señala también dos casos especiales de carácter sobrenatural y, por tanto, fuera del ámbito estrictamente filósofico, en que puede haber un conocimiento intuitivo de lo que todavía no existe, o en el que tiene lugar la intuición prescindiendo de la existencia.

Según el enunciado de la cuestión, podemos adivinar cuál es la respuesta de Atarrabia. Efectivamente, hay una diferencia entre la intuición y la abstracción, porque sólo la primera, y de modo ordinario, nos permite conocer la existencia. (Dejamos por el momento la posibilidad de que también abstractivamente se pueda conocer la existencia; esto es algo que Atarrabia discutirá más adelante.)

En el «videtur quod non» indica las razones que son contrarias a su propia tesis: parece que el intelecto humano «in terris» no tiene conocimiento intuitivo de las cosas; por ello, el conocimiento de la existencia debe ser por abstracción, como sucede en los demás casos. ${ }^{7}$

Sin embargo parece incompatible el conocimiento abstracto con el conocimiento de la existencia. Atarrabia echa mano de esta dificultad en la introducción misma de la cuestión. En efecto, lo que se conoce por abstracción no se conoce como «esto concreto», ya que conocer abstractivamente es conocer a partir de algo. El conocimiento abstracto es, de alguna manera, un conocimiento que requiere una mediación. La abstracción tiene lugar «desde algo», se abstrae «a partir de lo concreto». En el caso de que la existencia se conociera por abstracción, el hecho de ser conocida de este modo sería contrario a la razón propia de la existencia como tal, pues la existencia es concreta (en palabras de Atarrabia, «est ut haec»), y no parece que sea susceptible de ser abstraída. ${ }^{8}$ Así responde a la dificultad inicial, en el sentido de que intuición y abstracción se diferencian porque la existencia puede ser objeto conocido por intuición.

Pasa Atarrabia seguidamente a la exposición de qué es intuir y qué es abstraer. Se ocupa en la primera parte de la cuestión quodlibetal de decidir sobre este problema, a saber, distinguir cuáles sean las características del conocimiento intuitivo y cuáles las del

7 Pedro de Atarrabia, Quaestio Quodlibetale I, Utrum cognitio intuitiva et abstractiva distinguatur ab invicem [...], ed. Pío Sagüés, n. 1, p. 38: «Sed non cognoscitur exsistentia intuitive, quia talem cognitionem pro statu isto non habet intellectus de rebus: ergo abstractive».

8 Quodl. I, n. 2, p. 38: "Si ergo exsistentia cognoscitur, abstractive cognoscitur sub opposita ratione propriae rationi, quia exsistentia est ut "haec". Ergo exsistentia non potest cognosci abstractive». 
conocimiento abstracto en su respectiva relación con la existencia; es decir, si la existencia es objeto conocido por los dos tipos de noticia o si lo es sólo de uno de los dos.

Empieza Atarrabia estableciendo las tres condiciones de la intuición:

a) La intuición se refiere por sí y principalmente al objeto realmente existente, que es conocido como presente y existente. ${ }^{9}$

b) Ese objeto primum et per se de la intuición debe poseer la existencia actual en sí y no en algo que lo represente. ${ }^{10}$

c) Dicho objeto debe de estar presente en su existencia actual a la potencia cognoscitiva y ser clara e inmediatamente conocido. ${ }^{11}$

No obstante, Pedro de Atarrabia reconoce que las dos últimas condiciones no son intrínseca y formalmente necesarias para que el objeto de la noticia intuitiva sea conocido. Si fueran imprescindibles, dicho objeto estaría fuera del alcance de cualquier otro tipo de conocimiento. Pero no es así, ya que, como bien explica nuestro autor, aunque falten las características señaladas, ese objeto mismo puede ser conocido abstractivamente; luego, ya se ve que no son condiciones indispensables por parte del objeto conocido para que efectivamente sea conocido de una forma o de otra.

Lo expuesto en el Prólogo al Comentario de las Sentencias viene a reafirmar esta postura: lo que puede tener el ser-representado puede ser conocido en ese ser-representado, y ello aunque no esté presente realmente en su propia existencia actual al mismo cognoscente y aunque no tenga la existencia actual en alguna cosa exterior. Consta que aquello que es objeto de la noticia intutiva puede ser representado; así pues, el objeto de la noticia intuitiva puede serlo también de otro tipo de noticia, ya que no es contradictorio con el objeto conocido por intuición que sea representado en otro. ${ }^{12}$ Luego las dos últimas condiciones son condiciones anejas como razones formales del objeto; a pesar de ello, son necesariamente requeridas por parte del objeto en la intuición, ya que si carecemos de cualquiera de las dos, no es posible que tenga lugar la noticia intuitiva. ${ }^{13}$ En efecto, si el objeto de la intuición puede ser conocido por especie vicaria, cuánto más podrá ser conocido por abstracción, puesto que lo propio de la abstracción es conocer el objeto. En definitiva, el conocimiento abstracto se caracteriza, según Atarrabia, porque concierne a su objeto como presente en algo que lo representa, exista o no en algo exterior al cognoscente. ${ }^{1+}$

9 Quodl. I, n. 4, p. 39: «[...] respicit per se primum obiectum exsistens in re extra et ut praesens in actuali exsistentia propria in ratione obiecti».

10 Quodl. $I$, n. 4, p. 39: «[...] Illud primum et per se obiectum habeat esse exsistentiae actualis realiter extra in se, non in aliquo repraesentante ut cognito».

11 Quodl. I, n. 4, p. 39: «[...] Illud obiectum sit praesens in propria exsistentia actuali ipsi potentiae cognoscitivae ut obiectum inmediate et clare cognitum».

12 Pedro de Atarrabia, In I Sent., Pról, ed. cit., n. 11.

13 Quodl. I, n. 5, p. 39: «[...] sunt tamen conditiones annexae, et ita annexae quod necessario requisitae ex parte obiecti, quia aliter non esset intuitiva».

14 Quodl. I, n. 6, p. 39: «Notitia autem abstractiva est ista quae respicit suum obiectum ut praesens in aliquo repraesentativo in quo cognoscitur, sive exsistenes in re extra, sive non». 
Atarrabia no excluye el conocimiento abstracto de la existencia como otros autores de la misma época. Y no sólo se limita a aceptarla, sino que en esta cuestión quodlibetal distingue dos tipos de abstracción. El criterio de clasificación de estas dos noticias abstractas lo da la consideración de cuál sea el contenido cognoscitivo de cada una de ellas. El primer tipo de abstracción conoce la quididad o esencia, la cual es separada de la existencia en razón de objeto conocido. ${ }^{15}$ En el segundo modo, son conocidas esencia y existencia actual. En este caso, la esencia no es abstraída de la existencia, sino que ambas, esencia y existencia, son abstraídas de las condiciones requeridas en el objeto de la noticia intuitiva: se está refiriendo a que si algo es conocido a través de su «representación», lo es con independencia de que exista actualmente «fuera» del cognoscente. Tampoco es entonces necesario que ese objeto esté real y directamente presente, en su existencia propia, al intelecto que conoce.

El argumento que aduce Atarrabia para probar que si algo es conocido a través de una representación que incluya su esencia y su ser de existencia actual no es necesario que exista actualmente «fuera» del conocimiento, es el ejemplo de las criaturas presentes desde la eternidad en el entendimiento de quien les daría el ser. Éste es evidentemente un caso muy excepcional, que se separa del contexto gnoseológico que estamos tratando. Pero, puesto que Atarrabia le concede gran importancia argumentativa, lo veremos ahora. En efecto, Dios conoce las criaturas «ab aeterno» como existentes de manera clara e inmediata, aunque carezcan de existencia «exterior» a su creador y «conocedor». Tampoco, en ese caso, parece ser necesario, según Atarrabia, que el objeto esté presente en su propia existencia al mismo entendimiento que lo conoce. ${ }^{16}$

La tesis que Atarrabia estableció en la introducción de la cuestión quodlibetal, a saber que intuición y abstracción se diferencian en el modo de referirse a la existencia, parece estar ya firmemente asentada con este despliegue argumentativo. No obstante, todavía le quedan otras razones en apoyo de la dicha diferencia entre la intuición y la abstracción.

La existencia puede ser objeto conocido de igual modo por la abstracción que por la intuición. Puede ser también circunstancia requerida para que tenga lugar la noticia intuitiva. Sin embargo, el conocimiento abstracto sólo se puede referir a la existencia como a objeto conocido, mas no como algo necesario coexigido y requerido en el conocimiento. Con lo que establece la tan buscada diferencia entre la intuición y la abstracción en lo que a la existencia se refiere, esto es, la existencia no es condición requerida para el conocimiento abstracto del objeto. ${ }^{17}$

15 Quodl. I, n. 7. p. 39.

16 In I Sent., Pról. n. 14: «Similiter non est necesse, si aliquid cognoscitur aut representatur secundum essentiam et actualem exsistentiam et est exsisteens in re extra, quod, propter hoc, sit praesens ipsi cognoscenti in propria exsistentia, ut obiectum clare et inmediate cognitum, vel sit praesens in propria exsistentia ipsi repraesentanti».

17 Quodl. I, n. 8 y 9, p. 40: «Cognitio potest abstrahi, et potest concernere essentiam rei in effectu dupliciter: uno modo, quantum ad rationem obiecti cogniti; alio modo, quantum ad rationem alicuius necessario coexigiti. [...] Cognitio autem abstractiva solum concernere potest esse exsistentiae quantum ad rationem obiecti cogniti, non autem secundo modo». 


\section{PEDRO AUREOLO COMO CONTRADICTOR DE LA TESIS DE ATARRABIA}

Una vez que Atarrabia ha establecido claramente su argumentación a favor de la diferencia entre abstracción e intuición según la referencia de cada uno de estos dos tipos de noticia a la existencia, pasa al examen de la teoría de Pedro Aureolo, autor controvertido y sui generis donde los haya. Este pensador originario de Aquitania defendió ciertas teorías filosóficas bien avanzadas para su época, principios del siglo XIV, adelántandose incluso en algunos años a la síntesis ockamiana. ${ }^{18}$ Entre ellas destaca su postura en favor de la posibilidad de la intuición de lo inexistente.

Pedro Aureolo razona en contra de la relación estrecha de la existencia con la intuición, por la que la noticia intuitiva necesitaría que su objeto existiera realmente «fuera» del conocimiento. En efecto, Aureolo argumenta que se puede tener una intuición quiditativa de lo que no existe ahora. Y lo prueba con el hecho de que Dios desde la eternidad conoce intuitivamente la rosa como existente, ${ }^{19}$ a pesar de que es claro que la rosa no tuvo existencia sino en el momento de ser creada.

La segunda razón que da Aureolo es que Dios puede hacer que la visión que causa la blancura en el ojo se produzca efectivamente prescindiendo de la blancura. Se apoya en que los efectos que Dios puede realizar a través de una causa segunda, como sería que la blancura de un objeto exterior afectara a nuestros sentidos, pueden ser efectuados directamente por la Omnipotencia divina, sin mediación de causa segunda alguna. ${ }^{20}$ Por consiguiente, y según Aureolo, el hecho de que tenga lugar una intuición no significa que sea necesariamente causada por la existencia del objeto conocido de este modo.

Atarrabia rechaza que el caso del conocimiento intuitivo desde la eternidad de las criaturas por Dios sirva para explicar la posibilidad de la intuición de la quididad actualmente inexistente. En primer lugar, ya hemos visto como él considera que ese conocimiento que Dios tiene desde la eternidad es más bien un conocimiento mediato, abstracto, no un conocimiento intuitivo. Recuerda la primera condición necesaria que estableció al principio de la cuestión quodlibetal como característica de todo conocimiento intuitivo: la intuición se refiere por sí y principalmente a su objeto. El objeto primero y principal del conocimiento divino no son las criaturas sino el Verbo. La rosa no es objeto «primum et per se» del intelecto divino, sino objeto secundario, contenido de manera eminente en ese objeto primero y principal, y de este modo puede ser conocido intuitivamente aunque no exista «in re». ${ }^{21}$ Por tanto, en ese caso no hay propiamente intuición, según la definición establecida,

18 Cfr., José Antonio Merino, Historia de la filosofia franciscana, Madrid, 1993, pp. 286 y 387-388.

19 Quodl. I, n.10, p. 40: «Cognitio intuitiva potest esse quiditate quae non existit nunc: quia Deus ab aeterno cognovit rosam, ut exsistentem, intuitive».

20 Quodl. I, n. 10, p. 40: «Deus posset facere quod visio quam causat albedo in oculo, effective esset sine albedine, quia quidquid potest mediante causa secunda effective, potest sine illa».

21 Quodl. I, n. I1, p. 40: «Rosa et autem criatura aliae sunt secundarium obiectum, eminenter contentum in primo et per se obiecto; et ideo ab ipso potest cognosci intuitive in se, quamvis non exsistat in re». 
esto es, que la intuición se refiere principalmente y por sí a su objeto. Se trata, en todo caso, de una intuición sui generis.

El otro argumento de Aureolo, a saber, que Dios puede causar la visión del color, de la blancura, sin el objeto blanco, es matizada por Atarrabia. Lo visible es lo que se tiene en la visión $y$, desde ese punto de vista, lo visible puede ser efectivamente tenido sin la causa exterior. Pero el conocimiento de un color no puede quedarse ahí, puesto que la visión en sí misma carecería de color.

Para Atarrabia, en cambio, no puede haber intuición de un blanco inexistente. Dios sí puede hacer que se vea sin que se tenga algo visible. ${ }^{22}$ Dios puede causar la visión efectivamente sin lo blanco, pero no es posible que tal visión se refiera a algo determinado sin lo determinado. La visión no es blanca ni tiene color; sólo los objetos reales y existentes son coloreados. ${ }^{23}$ Según Atarrabia, se puede ver sin ver nada concreto, pero no se puede ver algo concreto si no existe tal concreto.

Un caso presentado por Duns Escoto en su Ordinatio (IV, d. 10, q. 9, n. 5) escapa a esta necesidad de la presencia del objeto existente para la intuición. Se trata de un ejemplo de índole sobrenatural. Escoto dice que Dios puede causar la visión del cuerpo de Cristo que está en la Eucaristía, pero es claro que en este caso la presencia eucarística no sirve para que el Cuerpo sea intuido. Dice que la visión es una forma absoluta, y por ello puede producirse sin depender de ningún tipo de presencia. De aquí deduce Escoto que la intuición puede ser sin objeto simpliciter. ${ }^{2+}$ Este último caso constituye una excepción a la condición general que Atarrabia estableció para que se produzca la noticia intuitiva.

También constituye una excepción el conocimiento que Dios tiene de las criaturas antes de la Creación. Las conoce en su existencia y su esencia aunque no existan in re. El conocimiento divino comprende lo que va a crear antes de crearlo; por lo tanto, prescinde claramente de la existencia de esas criaturas para acceder intuitivamente a ellas.

\section{CONCLUSIONES}

Intuición y abstracción se diferencian por su distinta forma de relacionarse con el objeto conocido. La intuición requiere la existencia real (es decir, extramental) del objeto conocido. Tal existencia es necesaria, pero en sí misma no es conocida. Se conoce el objeto como existente. La relación de la abstracción con la existencia es diferente. La abstracción prescinde generalmente de la existencia al conocer el objeto. Pero también se puede conocer

22 Quodl. I, n. 12, p. 41: «[...] non potest esse visio sine visibili, quia non est inconveniens duo absoluta se coexigere, sicut actus voluntatis coexigit actum prius intellectus».

23 Quodl. I, n. 12, p. 41: «Deus posset facere visionem sine visibili, quantum ad hoc quod visibile effective se habet ad visionem, sed non quantum ad rationem terminantis, cum non sit coloratum».

24 Quodl. I, n. 15, p. 41: «Ratio sua est quia visio est forma absoluta, et ideo potest fieri sine respectu praesentialitatis ad obiectum, vel sine quocumque respectu praesentialitatis» 
abstractamente la misma existencia. La existencia, pues, no es condición necesaria para la abstracción. En ningún modo es circunstancia necesaria para que la noticia abstracta tenga lugar.

Me parece que nos encontramos ante un autor que procede como si la existencia tuviera algunas de las caracteríscas de la esencia, como es la posibilidad de estar en la mente del cognoscente de modo abstracto, es decir, separado del objeto existente realmente, diciendo que esa existencia abstractamente conocida se diferencia de la existencia que es actualmente «extra». Aplica esta noción directamente a las criaturas contenidas en la mente divina desde la eternidad; las conocía como existentes cuando todavía no existían fuera del conocimiento que Dios tenía de ellas. Y para Atarrabia es el ejemplo patente de que algo similar ocurre en el conocimiento que conviene al intelecto humano.

Por consiguiente, la existencia se caracteriza porque no se identifica con la esencia. Ya hemos dicho que no es objeto coexigido por la noticia abstracta; pero cuando Atarrabia clasifica los dos tipos de abstracción dice que la existencia puede ser objeto del conocimiento abstracto. Considera, pues, esta «existencia conocida» como teniendo en la mente del cognoscente cierta «entidad» distinta de la existencia que tiene la cosa exterior al conocimiento. Por todo ello, creo que se puede decir que Atarrabia acepta una cierta formalización de la existencia.

Ana Azanza

Instituto de Historia de la Iglesia

Universidad de Navarra

E-31080 Pamplona 